\title{
Natural modes for the analysis of optical bistability and laser instability
}

\author{
Lloyd W. Hillman, Robert W. Boyd, and C. R. Stroud, Jr. \\ The Institute of Optics, University of Rochester, Rochester, New York 14627
}

Received April 26, 1982

\begin{abstract}
A self-consistent description is given for the propagation of a weak optical probe beam through a homogeneously broadened two-level medium in the presence of a strong, collinear, near-resonant pump beam. The propagation of the probe beam is given in terms of bichromatic natural modes, which contain frequency components symmetrically displaced from that of the pump beam. The two frequency components have a definite relative amplitude and phase, which are determined solely by the frequency detunings of the optical fields from the atomic resonance and by the intensity of the pump field. A simple method for calculating these natural modes is derived, and their importance in describing nearly degenerate four-wave mixing, optical bistability, and the stability of homogeneously broadened ring lasers is discussed.
\end{abstract}

The propagation of a weak, monochromatic probe beam, tuned near a resonance frequency of an atomic vapor, is profoundly influenced by the presence of a strong monochromatic pump field that is nearly resonant with the same transition. A probe field injected into the medium with a frequency detuned by an amount $\delta \nu$ from the frequency $\omega_{0}$ of the pump field will be strongly coupled to another frequency component of the field, symmetrically detuned from $\omega_{0}$ by the amount $-\delta \nu$. The coupling between these two monochromatic-field components is often so strong that the problem is better described in terms of its natural modes, which are linear combinations of these two side bands with well-defined relative amplitudes and phases.

In this Letter we derive the form of these natural modes and show that in general (for a noninverted medium) one mode will experience loss while the other may experience gain. A probe field will, on propagation through the medium, develop into one of these natural modes. Previous work has calculated the induced polarization in the medium for assumed relative amplitudes and phases of the two coupled frequency components of the probe field. The probe gain and dispersion are calculated subject to this arbitrary ansatz. ${ }^{1,2}$ Such calculations are appropriate only for propagation through a distance short compared with the Beer length of the medium.

Our analysis is applicable to resonantly enhanced, nearly degenerate four-wave mixing in atomic vapors, ${ }^{2}$ in which the coupling between the two side bands has been observed. ${ }^{1,3}$ It is also applicable to the treatment of bistability involving a two-level medium placed inside a resonant ring cavity ${ }^{4}$ and to the stability analysis of an unidirectional homogeneously broadened ring laser far above threshold. ${ }^{5}$

We obtain the natural modes of the probe field using self-consistent semiclassical radiation theory: the medium is described by the Bloch equations, and the field is described by the reduced Maxwell equations. ${ }^{6}$ By self-consistency we mean that we solve these equa- tions simultaneously, including the effect both of the field on the atom and of the atom on the field.

The field is assumed to be a plane wave propagating in the $z$ direction and can be written in the form

$$
\begin{aligned}
E(z, t)= & 2 \operatorname{Re}\left[\tilde{\mathscr{E}}_{0}(z) \exp i \omega_{0} t+\delta \tilde{E}_{+}(z) \exp i\left(\omega_{0}+\delta \nu\right) t\right. \\
& \left.+\delta \tilde{\mathscr{E}}-(z) \exp i\left(\omega_{0}-\delta \nu\right) t\right] \\
\equiv & 2 \operatorname{Re}\left\{\tilde{\mathscr{E}}(z, t) \exp i\left[\omega_{0} t-k_{0} z+\phi_{0}(z)\right]\right\}
\end{aligned}
$$

where

$$
\begin{aligned}
\tilde{\mathscr{E}}(z, t)= & \mathscr{E}_{0}(z)+\delta \tilde{\mathscr{E}}(z, t), \\
\delta \tilde{\mathscr{E}}(z, t)= & \delta \mathscr{E}(z)[\cos \psi \exp i \Phi \\
& +\sin \psi \exp i(\Phi+\theta)],
\end{aligned}
$$

and

$$
\Phi=\delta \nu t-\frac{\delta \nu}{c} z+\phi_{1}(z)
$$

The slowly varying complex amplitude of the total field is denoted here as $\tilde{\mathscr{E}}(z, t)=\mathscr{E}^{\prime}(z, t)+i \mathscr{E}^{\prime \prime}(z, t)$ (we consistently use the tilde to denote complex quantities), and the magnitude of the vacuum wave vector of the pump field is denoted as $k_{0}=\omega_{0} / c$. The phase variation of the pump field that is due to the dispersion of the medium is denoted as $\phi_{0}(z)$ and is defined such that the pump-field amplitude $\mathscr{E}_{0}(z)$ is everywhere a real quantity. The probe-field amplitude is denoted as $\delta \tilde{E}(z, t)=\delta \mathscr{E}^{\prime}(z, t)+i \delta \mathscr{E}^{\prime \prime}(z, t)$. The phase of the probe field varies spatially relative to that of the pump field because of their vacuum wave-vector difference $\delta \nu / c$ and as $\phi_{1}(z)$ because of the dispersion of the medium. At fixed $z, \delta \tilde{\mathscr{E}}(z, t)$ periodically sweeps out an elliptical orbit in the complex $\tilde{E}$ plane, as shown in Fig. 1. Finally, we define the natural modes of the probe field as the linear combinations of the two side bands for which the amplitude ratios $\tan \psi$ and relative phases $\theta$ do not vary with propagation distance $z$.

We assume that the components of the Bloch vector can be decomposed into a contribution that is due to the pump and a small perturbation that is due to the probe so that $u(z, t)=u_{0}(z)+\delta u(z, t), \nu(z, t)=\nu_{0}(z)+\delta \nu(z$, 


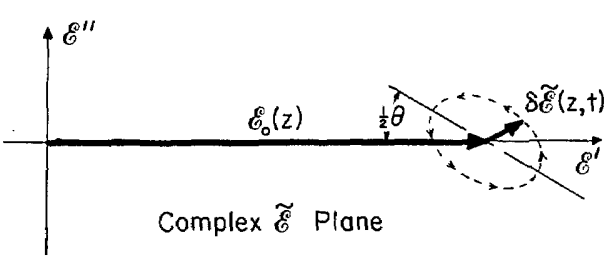

Fig. 1. The complex amplitude of the total electric field decomposed into the pump amplitude $\mathscr{E}_{0}(z)$ and the probe amplitude $\delta \tilde{E}(z, t)$. In the complex $\tilde{\mathscr{E}}$ plane, $\mathscr{E}_{0}(z)$ lies along the real axis while $\delta \tilde{E}(z, t)$ revolves periodically around an ellipse.

$t)$, and $w(z, t)=w_{0}(z)+\delta w(z, t)$. If we substitute this perturbation expansion into the Bloch equations and neglect terms quadratic in the perturbation, we arrive at a linear set of equations for the perturbed Bloch vector:

$$
\begin{aligned}
\frac{\mathrm{d}}{\mathrm{d} t}\left(\begin{array}{c}
\delta u \\
\delta v \\
\delta w
\end{array}\right)= & \left(\begin{array}{ccc}
-\frac{1}{T_{2}} & -\Delta & 0 \\
+\Delta & -\frac{1}{T_{2}} & \kappa \mathscr{E}_{0} \\
0 & -\kappa \mathscr{E}_{0} & -\frac{1}{T_{1}}
\end{array}\right)\left(\begin{array}{c}
\delta u \\
\delta \nu \\
\delta w
\end{array}\right) \\
& +\left(\begin{array}{c}
-\kappa w_{0} \delta \mathscr{E}^{\prime \prime} \\
\kappa w_{0} \delta \mathscr{E}^{\prime} \\
\kappa u_{0} \delta \mathscr{E}^{\prime \prime}-\kappa \nu_{0} \delta \mathscr{E}^{\prime}
\end{array}\right)
\end{aligned}
$$

The detuning of the pump laser from the atomic resonance is denoted $\Delta=\omega_{a}-\omega_{0} K=2 \mathrm{~d} / \hbar$ is proportional to the transition dipole moment, and $T_{1}$ and $T_{2}$ are, respectively, the longitudinal and transverse relaxation times. ${ }^{7}$

In a similar fashion we find the reduced Maxwell equations for the probe field coupled to the perturbed Bloch vector:

$$
\begin{aligned}
\left(\frac{\partial}{\partial z}+\frac{1}{c} \frac{\partial}{\partial t}\right)\left(\begin{array}{l}
\delta \mathscr{E}^{\prime} \\
\delta \mathscr{E}^{\prime \prime}
\end{array}\right)= & \frac{\partial \phi_{0}}{\partial \mathrm{z}}\left(\begin{array}{rr}
0 & 1 \\
-1 & 0
\end{array}\right)\left(\begin{array}{l}
\delta \mathscr{E}^{\prime} \\
\delta \mathscr{E}^{\prime \prime}
\end{array}\right) \\
& +\frac{\alpha}{2 \kappa T_{2}}\left(\begin{array}{r}
\delta \nu \\
-\delta u
\end{array}\right) .
\end{aligned}
$$

The constant $\alpha$ is the unsaturated reciprocal Beer absorption length at line center. The propagation of the probe field is described by the self-consistent solution of Eqs. (5) and (6).

Equation (5) is a driven, linear differential equation, and thus its harmonic particular solution can be found exactly for arbitrary probe-field amplitudes and the results substituted into Eq. (6). The most convenient way to carry out this procedure is to introduce a complex representation: $\delta \mathscr{E}^{\prime}(z, t)$ and $\delta \mathscr{E}^{\prime \prime}(z, t)$ are taken as the real parts of complex amplitudes oscillating at the harmonic frequency $\delta \nu$,

$$
\delta \mathscr{E}^{\prime}(z, t)=\operatorname{Re}\left[\delta \tilde{E}^{\prime}(z) \exp i\left(\delta \nu t-\frac{\delta \nu}{c} z\right)\right]
$$

$$
\delta \mathscr{E}^{\prime \prime}(z, t)=\operatorname{Re}\left[\delta \tilde{\mathscr{E}}^{\prime \prime}(z) \exp i\left(\delta \nu t-\frac{\delta \nu}{c} z-\frac{\pi}{2}\right)\right] .
$$

This representation is identical with that introduced in the theory of polarized light in which the polarized field is represented by the complex two-dimensional Jones vector. ${ }^{8}$ The complex amplitudes then satisfy the equation

$$
\begin{aligned}
\frac{\partial}{\partial z}\left(\begin{array}{l}
\delta \tilde{\mathscr{E}}^{\prime}(z) \\
\tilde{\delta \tilde{E}^{\prime \prime}}(z)
\end{array}\right)= & \frac{\alpha w_{0}(z)}{2 \tilde{\Gamma}(z)\left[1+\left(\Delta T_{2}\right)^{2}\right]} \\
& \times\left(\begin{array}{ll}
\tilde{b}_{11}(z) & \tilde{b}_{12}(z) \\
\tilde{b}_{21}(z) & \tilde{b}_{22}(z)
\end{array}\right)\left(\begin{array}{l}
\delta \tilde{\mathscr{E}}^{\prime}(z) \\
\delta \tilde{\mathscr{E}}^{\prime \prime}(z)
\end{array}\right),
\end{aligned}
$$

where we have introduced the following coefficients:

$$
\begin{aligned}
\tilde{b}_{11}(z) & =\left(1+i \delta \nu T_{2}\right)\left[1+\left(\Delta T_{2}\right)^{2}-\frac{I_{0}(z)}{1+i \delta \nu T_{1}}\right], \\
\tilde{b}_{12}(z) & =\Delta T_{2} \delta \nu T_{2}\left(2+i \delta \nu T_{2}\right), \\
\tilde{b}_{21}(z) & =\Delta T_{2}\left(2+i \delta \nu T_{2}\right)\left[\delta \nu T_{2}-\frac{i I_{0}(z)}{1+i \delta \nu T_{1}}\right], \\
\tilde{b}_{22}(z) & =\left(1+i \delta \nu T_{2}\right)\left[1+\left(\Delta T_{2}\right)^{2}\right]+\frac{I_{0}(z)}{1+i \delta \nu T_{1}}, \\
\tilde{\Gamma}(z) & =\left(1+i \delta \nu T_{2}\right)^{2}+\left(\Delta T_{2}\right)^{2}+\left(\frac{1+i \delta \nu T_{2}}{1+i \delta \nu T_{1}}\right) I_{0}(z),
\end{aligned}
$$

the normalized population difference

$$
w_{0}(z)=w_{\mathrm{eq}} \frac{1+\left(\Delta T_{2}\right)^{2}}{1+\left(\Delta T_{2}\right)^{2}+I_{0}(z)}
$$

and the dimensionless intensity of the pump field

$$
I_{0}(z)=\kappa^{2} T_{1} T_{2} \mathscr{E}_{0}^{2}(z) .
$$

For a pump field detuned far from resonance or sufficiently strong to saturate the atomic absorption, the spatial variation of $I_{0}(z)$ is small, and the coefficients in Eq. (8) are nearly constant. In this Letter we limit our discussion to a mean-field or nondepleted-pump approximation by taking $I_{0}$ to be constant, and therefore the solution to Eq. (8) will satisfy

$$
\begin{aligned}
\frac{\partial}{\partial z}\left(\begin{array}{l}
\delta \tilde{\mathscr{E}}^{\prime}(z) \\
\delta \tilde{\mathscr{E}}^{\prime \prime}(z)
\end{array}\right) \rightarrow & \left(1 / 2 \alpha_{1}+i \frac{\partial \phi_{1}}{\partial z}\right)\left(\begin{array}{l}
\delta \tilde{\mathscr{E}}^{\prime}(z) \\
\delta \tilde{\mathscr{E}^{\prime \prime}}(z)
\end{array}\right) \\
& \equiv 1 / 2 \tilde{\alpha}_{1}\left(\begin{array}{l}
\delta \tilde{\tilde{E}^{\prime}}(z) \\
\delta \tilde{\mathscr{E}}^{\prime \prime}(z)
\end{array}\right) .
\end{aligned}
$$

The eigenvalue equation that results from Eqs. (8) and (9) defines the natural modes of the probe field. The eigenvalues

$$
\begin{aligned}
{ }_{1 / 2} \tilde{\alpha}_{ \pm}= & \frac{\alpha w_{0}}{4 \tilde{\Gamma}\left[1+\left(\Delta T_{2}\right)^{2}\right]}\left\{\left(\tilde{b}_{11}+\tilde{b}_{22}\right)\right. \\
& \pm\left[\left(\tilde{b}_{11}-\tilde{b}_{22}\right)^{2}+4 \tilde{b}_{12} \tilde{b}_{21}\right]^{1 / 2}
\end{aligned}
$$

are the complex absorption coefficients of the natural modes. The associated eigenvectors represent what will be called the plus and minus modes.

To illustrate the natural-mode solution, four cases are graphically displayed in Fig. 2. Case 1 assumes a pump-field detuning of $\Delta T_{2}=3$ in the limit that the pump field has zero intensity. Case 2 is a resonant pump field $\left(\Delta T_{2}=0\right)$ with Rabi frequency $\kappa T_{2} \mathscr{E}_{0}=8$ 


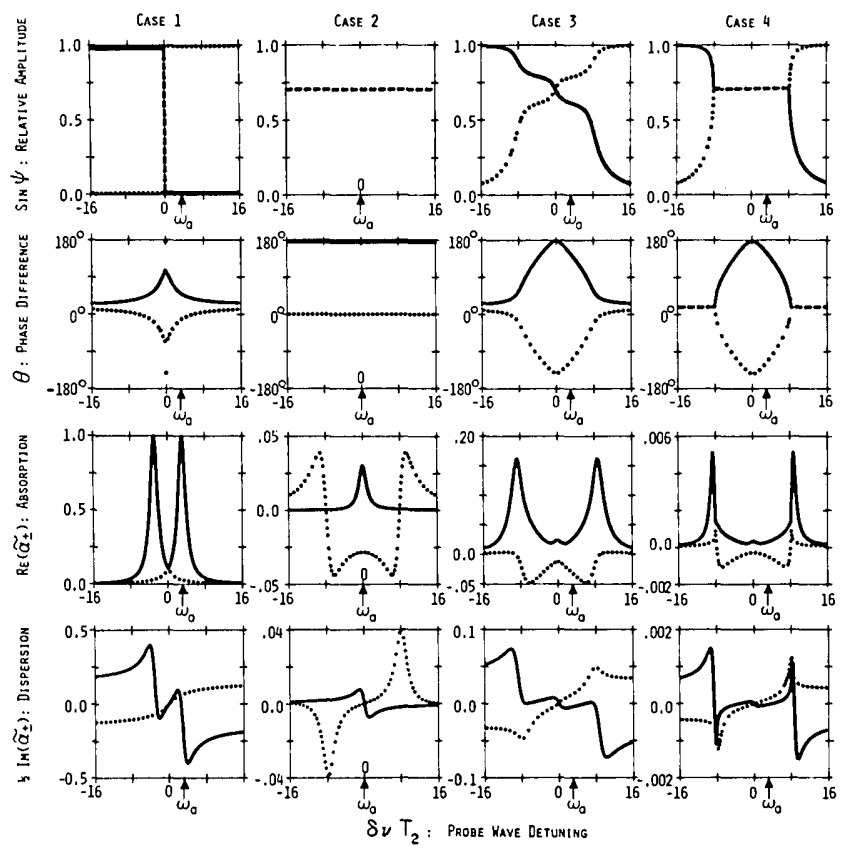

Fig. 2. Graphically illustrated are the relative amplitude and phase difference of the two side bands and the absorption and dispersion coefficients as functions of the probe-wave detuning $\delta \nu T_{2}$ for the natural-mode solution. The solid and dotted lines are for the plus and minus modes, respectively. The four cases illustrated are (1) $\kappa T_{2} \mathscr{E}_{0}=0$ and $\Delta T_{2}=3 ;$ (2) $\kappa T_{2} \mathscr{E}_{0}=8, \Delta T_{2}=0$, and $T_{2} / T_{1}=2 ;(3) \kappa T_{2} \mathscr{E}_{0}=8, \Delta T_{2}=3$, and $T_{2} / T_{1}=2$; and (4) $\kappa T_{2} \mathscr{E}_{0}=8, \Delta T_{2}=3$, and $T_{2} / T_{1}=$ 0.02 .

and purely radiative broadening $\left(T_{2} / T_{1}=2\right)$. Cases 3 and 4 treat a pump field with Rabi frequency $\kappa T_{2} \mathscr{E}_{0}=$ 8 and detuning $\Delta T_{2}=3$. Case 3 treats purely radiative broadening, whereas Case $4, T_{2} / T_{1}=0.02$, corresponds to rapid collisional dephasing. In all the figures, the plus mode is shown by the solid line and the minus mode by the dotted line.

In the first row of Fig. 2 we have graphed the quantity $\sin \psi$ as a function of probe detuning $\Delta T_{2}$. From Eq. (3) we see that $\sin \psi$ is the normalized amplitude of the $-\delta \nu$ side band in the coupled solution. The phase difference $\theta$ between these two side bands is plotted in the second row. Case 1 shows that for a zero-intensity pump field the natural modes are simply the two uncoupled side bands. For Case 2, the minus and plus natural modes are linear combinations of the two side bands with equal magnitude. For the minus mode the side bands are in phase; for the plus mode they are $180^{\circ}$ out of phase. The natural modes of the probe field for the exactly resonant pump are therefore the AM and FM modes. Cases 3 and 4 illustrate that strong coupling occurs between the two side bands whenever $|\delta \nu|$ $\lesssim\left(\Delta^{2}+\kappa^{2} \mathscr{E}_{0}^{2}\right)^{1 / 2}$. This coupling is enhanced by strong collisional dephasing. The phase-difference curves for the plus and minus modes are symmetric about and asymptotically approach the value $\theta=\operatorname{cotan}^{-1}\left(\Delta T_{2}\right)$.

In the third row, the real part of the complex absorption coefficient (which gives rise to absorption) is plotted as a function of the probe-wave detuning. One half of the imaginary part of the complex absorption coefficient (which gives rise to dispersion) is displayed in the fourth row. Both coefficients are normalized by the Beer absorption length $\alpha$.

For an absorber, the minus mode may have gain for probe detunings $|\delta \nu| \lesssim\left(\kappa^{2} \mathscr{E}_{0}^{2}+\Delta^{2}\right)^{1 / 2}$, as illustrated in Cases 2-4. The steady-state, bistable transmission of a cw-driven ring cavity containing the two-level absorber will become unstable if the gain of a minus mode is greater than the round-trip loss. Cavity boundary conditions, including the probe-field dispersion, must also be met. Gain occurs for the minus mode only when the two side bands are strongly coupled. This explains the appearance of both side bands in experiments on nearly degenerate four-wave mixing in extended media.

For a pump laser detuned from resonance, the plus mode has two or three absorption peaks. One may be at the laser frequency while the other two are symmetrically displaced from the pump frequency by approximately the generalized Rabi frequency, i.e., $|\delta \nu| \simeq$ $\left(\kappa^{2} \mathscr{E}_{0}^{2}+\Delta^{2}\right)^{1 / 2}$. The minus root also has an absorption peak at the generalized Rabi frequency. The absorption of the plus mode at the central peak is equal to the saturated absorption coefficient of the pump field. For an inverted medium, these absorption peaks become regions of gain. A homogeneously broadened laser cannot run in a single mode if the gain near the Rabi side bands exceeds that of the central peak and if the natural mode in question satisfies the cavity-boundary conditions. This may happen for either the minus (Case 2) or the plus (Case 3 ) mode or both (Case 4).

In conclusion, we have shown that the self-consistent treatment of a probe beam propagating through a driven two-level medium is described in terms of bichromatic natural modes. The natural modes permit a simple interpretation of results in nearly degenerate four-wave mixing and are the proper modes for analyzing the stability of homogeneously broadened ring lasers and bistable ring cavities.

\section{References}

1. B. Senitzky, G. Gould, and S. Cutler, Phys. Rev. 130, 1460-1465 (1963).

2. T. Y. Fu and M. Sargent III, Opt. Lett. 4, 366-368 (1979); D. J. Harter and R. W. Boyd, IEEE J. Quantum Electron. QE-16, 1126-1131 (1980).

3. D. J. Harter, P. Narum, M. G. Raymer, and R. W. Boyd, Phys. Rev. Lett. 46, 1192-1195 (1981).

4. R. Bonifacio and L. A. Lugiato, Opt. Commun. 19, 172-176 (1976).

5. H. Risken and K. Nummedal, Phys. Lett. A26, 275-276 (1968); S. T. Hendow and M. Sargent III, Opt. Commun. 40, 385-390 (1982).

6. S. L. McCall, Phys. Rev. A 9, 1515-1523 (1974).

7. Our notation follows as closely as possible that of the text by L. Allen and J. H. Eberly, Optical Resonance and Two-Level Atoms (Wiley, New York, 1975).

8. The two components of the Jones polarization vector represent the complex amplitudes of the horizontal and vertical linear polarizations. Under the complex representation presented here, the two field components $\delta \mathscr{E}^{\prime}(z)$ and $\delta \tilde{\mathscr{E}}^{\prime \prime}(z)$ represent the complex amplitude contributions of the AM and FM modes, respectively. See W. A. Shurcliff, Polarized Light (Harvard U. Press, Cambridge, Mass., 1962). 